\title{
A LÓPEZ-ESCOBAR THEOREM FOR METRIC STRUCTURES, AND THE TOPOLOGICAL VAUGHT CONJECTURE
}

\author{
SAMUEL COSKEY AND MARTINO LUPINI
}

\begin{abstract}
We show that a version of López-Escobar's theorem holds in the setting of model theory for metric structures. More precisely, let $\mathbb{U}$ denote the Urysohn sphere and let $\operatorname{Mod}(\mathcal{L}, \mathbb{U})$ be the space of metric $\mathcal{L}$-structures supported on $\mathbb{U}$. Then for any Iso(U) invariant Borel function $f: \operatorname{Mod}(\mathcal{L}, \mathbb{U}) \rightarrow[0,1]$, there exists a sentence $\phi$ of $\mathcal{L}_{\omega_{1} \omega}$ such that for all $M \in \operatorname{Mod}(\mathcal{L}, \mathbb{U})$ we have $f(M)=\phi^{M}$. This answers a question of Ivanov and Majcher-Iwanow. We prove several consequences, for example every orbit equivalence relation of a Polish group action is Borel isomorphic to the isomorphism relation on the set of models of a given $\mathcal{L}_{\omega_{1}} \omega$-sentence that are supported on the Urysohn sphere. This in turn provides a model-theoretic reformulation of the topological Vaught conjecture.
\end{abstract}

\section{§1. BACKGRound AND STATEMENT OF MAIN RESUlT}

A well-known theorem of López-Escobar [LE] says roughly that every Borel class of countable structures can be axiomatized by a sentence in the logic where countable conjunctions and disjunctions are allowed. The theorem has been generalized to apply to wider classes of structures, using sentences from a variety of logics (see for example $[\mathrm{T}, \mathrm{V}]$ ).

To state López-Escobar's theorem more precisely, let $\mathcal{L}$ be a countable first-order language consisting of the relational symbols $\left\{R_{i}\right\}$ where each $R_{i}$ has arity $n_{i}$. The space $\operatorname{Mod}(\mathcal{L})$ of countably infinite $\mathcal{L}$-structures is given by

$$
\operatorname{Mod}(\mathcal{L})=\prod \mathcal{P}\left(\mathbb{N}^{n_{i}}\right)
$$

and we note it is compact in the product topology. The space carries a natural $S_{\infty}$-action by left-translation on each factor, and the $S_{\infty}$-orbits are precisely the isomorphism classes.

Next, recall that $\mathcal{L}_{\omega_{1} \omega}$ denotes the extension of first-order logic in which countable conjunctions and disjunctions are allowed (formulas are still only allowed to have finitely many free variables). If $\phi$ is a sentence of $\mathcal{L}_{\omega_{1} \omega}$ then the subset $\operatorname{Mod}(\phi) \subset \operatorname{Mod}(\mathcal{L})$ consisting just of the models of $\phi$ is clearly $S_{\infty}$-invariant (isomorphism invariant), and it is easy to see that

2000 Mathematics Subject Classification. Primary 03C95, 03E15; Secondary 54E50.

Key words and phrases. Model theory for metric structures, infinitary logic, Polish group action, Urysohn sphere.

The second author is supported by the York University Elia Scholars Program and by the York University Graduate Development Fund. Much of this work was completed during a visit of the second author to Boise State University in April 2014. The hospitality of the BSU Mathematics Department is gratefully acknowledged. 
it is Borel. López-Escobar's theorem states that the converse holds, that is if $A \subset \operatorname{Mod}(\mathcal{L})$ is Borel and $S_{\infty}$-invariant, then there exists a sentence $\phi$ of $\mathcal{L}_{\omega_{1} \omega}$ such that $A=\operatorname{Mod}(\phi)$.

López-Escobar's theorem has numerous applications. For instance, the Vaught conjecture for $\mathcal{L}_{\omega_{1} \omega}$ states that any set $\operatorname{Mod}(\phi)$ contains either just countably many nonisomorphic structures or perfectly many nonisomorphic structures (we will make this precise in the next section). More generally, the topological Vaught conjecture for $S_{\infty}$ states that any Borel action of $S_{\infty}$ has either countably or perfectly many orbits. It follows from López-Escobar's theorem together with some standard facts about Polish group actions that the topological Vaught conjecture for $S_{\infty}$ is equivalent to the Vaught conjecture for $\mathcal{L}_{\omega_{1} \omega}$.

In [IMI], the authors generalize numerous properties of the space of countable discrete structures to spaces of separable complete metric structures. They ask whether a version of López-Escobar's theorem holds in the metric context. In this article we confirm that the natural generalization of López-Escobar's theorem to spaces of metric structures supported on the Urysohn sphere holds. We use this result to derive several corollaries, including an equivalence between the topological Vaught conjecture and a Vaught conjecture for metric structures.

Before stating our result precisely, we begin with a brief introduction to logic for metric structures. For a full account of this fruitful area, we refer the reader to [BYBHU]. As in first-order logic, in logic for metric structures a language $\mathcal{L}$ consists of function symbols $f$ and relation symbols $R$, each with a finite arity $n_{f}$ or $n_{R}$. Additionally, to each function symbol $f$ or relation symbol $R$ there is a corresponding modulus of continuity $\varpi_{f}$ or $\varpi_{R}: \mathbb{R}_{+} \rightarrow \mathbb{R}_{+}$which is continuous and vanishes at 0 . Now, an $\mathcal{L}$-structure $M$ consists of a support, which is a complete metric space (also denoted $M$ ), together with interpretations of the function and relation symbols of $\mathcal{L}$. That is, for each function symbol $f$ we have a function $f^{M}: M^{n_{f}} \rightarrow M$ which is uniformly continuous with modulus of continuity $\varpi_{f}$ :

$$
d\left(f^{M}(\bar{a}), f^{M}(\bar{b})\right) \leq \varpi_{f}(d(\bar{a}, \bar{b}))
$$

(Here, as with all finite products, we consider the maximum metric on $M^{n_{f}}$.) Similarly, for each relation symbol $R$ we have a function $R^{M}: M^{n_{R}} \rightarrow[0,1]$ which is uniformly continuous with modulus $\varpi_{R}$.

We now briefly discuss the syntax of logic for metric structures. Given a language $\mathcal{L}$, we define the formulas of $\mathcal{L}$ as follows. The terms and atomic formulas are defined in the usual way, except that instead of the $=$ symbol, we include a binary function symbol $d$ which is always interpreted as the metric. The connectives are continuous functions $h:[0,1]^{n} \rightarrow$ $[0,1]$, so if $\phi_{0}, \ldots, \phi_{n-1}$ are formulas and $h$ is such a function then $h\left(\phi_{0}, \ldots, \phi_{n-1}\right)$ is a formula. The quantifiers are sup and inf, so if $\phi$ is a formula and $x$ is a variable, then $\inf _{x} \phi$ and $\sup _{x} \phi$ are formulas.

For our generalization of López-Escobar's theorem, we will use the infinitary language $\mathcal{L}_{\omega_{1} \omega}$ in the metric setting as defined in [BYI, Theorem 1.1]. (Other infinitary logics for metric structures are studied in [E], and [S].) Here, if $\phi_{n}$ is a sequence of $\mathcal{L}_{\omega_{1} \omega}$-formulas (with 
finitely many free variables among them all), then $\inf _{n} \phi_{n}$ and $\sup _{n} \phi_{n}$ are $\mathcal{L}_{\omega_{1} \omega}$-formulas provided that the sequence of uniform continuity moduli is itself uniformly bounded. Every $\mathcal{L}_{\omega_{1} \omega}$-formula $\phi$ has a corresponding modulus of continuity $\varpi_{\phi}$, defined by recursion on the complexity of $\phi$.

Note that if $\phi$ is a sentence of $\mathcal{L}_{\omega_{1} \omega}$ and $M$ is an $\mathcal{L}$-structure, then $\phi^{M}$ is naturally interpreted as an element of $[0,1]$. Intuitively the value 0 means that $\phi$ is certainly true in $M$, and larger values give shades of grey truth. Thus the evaluation map $M \mapsto \phi^{M}$ is an example of a grey set.

Grey sets, originally named graded sets, were introduced in [BYM] and used extensively in [IMI]. If $X$ is a topological space then $A$ is said to be a grey subset of $X$, written $A \sqsubseteq X$, if $A$ is a function $X \rightarrow[0,1]$. The sets $A_{<r}=\{x \in X \mid A(x)<r\}$ and $A_{\leq r}=\{x \in$ $X \mid A(x) \leq r\}$ are called the level sets of $A$. The terminology of grey set arises from the idea that asking whether $x \in A_{<r}$ is not a black-and-white question but rather one which depends on the parameter $r \in[0,1]$.

It is possible to generalize a number of concepts from point-set topology and descriptive set theory to grey sets. For example, $A \sqsubseteq X$ is said to be open if $A_{<r}$ is open for all $r$ ( $A$ is upper semicontinuous), and closed if $A_{\leq r}$ is closed for all $r$ ( $A$ is lower semicontinuous); see [BYM, Definition 1.4]. More generally one can define the Borel classes $\boldsymbol{\Sigma}_{\alpha}^{0}$ and $\boldsymbol{\Pi}_{\alpha}^{0}$ of Borel grey subsets of $X$ by induction on $\alpha \in \omega_{1}$ as in [IMI, Section 2.1]:

○ $A \in \Sigma_{1}^{0}$ iff $A$ is an open grey subset of $X$;

$\circ A \in \boldsymbol{\Pi}_{\alpha}^{0}$ iff $1-A \in \boldsymbol{\Sigma}_{\alpha}^{0}$; and

○ $A \in \boldsymbol{\Sigma}_{\alpha}^{0}$ iff $A=\inf _{n} A_{n}$ where $A_{n} \in \bigcup_{\beta<\alpha} \boldsymbol{\Pi}_{\beta}^{0}$.

We then say $A \sqsubseteq X$ is Borel if it belongs to $\Sigma_{\alpha}^{0}$ for some $\alpha<\omega_{1}$, and by [K, Theorem 24.3] $A$ is Borel iff it is Borel as a function $X \rightarrow[0,1]$. Continuing up the projective hierarchy, a grey subset $A$ of $X$ is analytic if there is a Borel grey subset $B \sqsubseteq X \times Y$ for some Polish space $Y$ such that $A=\inf _{y} B$, i.e., for every $x \in X$

$$
A(x)=\inf _{y \in Y} B(x, y)
$$

It is not difficult to verify that $A$ is analytic iff the level sets $A_{<r}$ are analytic for all $r \in \mathbb{Q}$. Similarly, $A$ is coanalytic iff $1-B$ is analytic, or equivalently $B_{\leq r}$ is coanalytic for every $r \in \mathbb{Q}$.

We now return to our motivating example of the evaluation map for a given sentence. Fix a separable complete metric space $Y$, and denote by $\operatorname{Iso}(Y)$ the group of isometries of $Y$ (it is a Polish group with respect to the topology of pointwise convergence). As with countable discrete structures, there is naturally a space $\operatorname{Mod}(\mathcal{L}, Y)$ of $\mathcal{L}$-structures having $Y$ as support:

$$
\operatorname{Mod}(\mathcal{L}, Y)=\prod \operatorname{Unif}_{\varpi_{f}}\left(Y^{n_{f}}, Y\right) \times \prod \operatorname{Unif}_{\varpi_{R}}\left(Y^{n_{R}},[0,1]\right)
$$


Here $\operatorname{Unif}_{\varpi}(A, B)$ denotes the space of $\varpi$-uniformly continuous functions from $A$ to $B$ with the topology of pointwise convergence. Then $\operatorname{Mod}(\mathcal{L}, Y)$ is easily seen to be a Polish Iso $(Y)$-space with respect to the natural action of $\operatorname{Iso}(Y)$. Now if $\phi$ is an $\mathcal{L}_{\omega_{1} \omega}$-sentence we can define the evaluation map $E_{\phi} \sqsubseteq \operatorname{Mod}(\mathcal{L}, Y)$ by

$$
E_{\phi}(M)=\phi^{M} \text {. }
$$

More generally if $\phi(\bar{x})$ is an $\mathcal{L}_{\omega_{1} \omega}$-formula with $n$-free variables we can define the evaluation $\operatorname{map} E_{\phi} \sqsubseteq \operatorname{Mod}(\mathcal{L}, Y) \times Y^{n}$ by

$$
E_{\phi}(M, u)=\phi^{M}(u)
$$

It is not difficult to verify that the evaluation function $E_{\phi}$ for a formula of $\mathcal{L}_{\omega_{1} \omega}$ is always Borel (see Proposition 3.1).

This brings us to our main result, which asserts that any grey subset of $\operatorname{Mod}(\mathcal{L}, \mathbb{U})$ which is Borel and $\operatorname{Iso}(\mathbb{U})$-invariant arises as an evaluation $E_{\phi}$. Here $\mathbb{U}$ denotes the Urysohn sphere, which is the unique metric space that is separable, complete, ultrahomogeneous, with metric bounded by 1, and which contains an isometric copy of any other separable metric space with metric bounded by 1 . A survey of the remarkable properties of the Urysohn sphere can be found in [M3].

Theorem 1.1. For every $\operatorname{Iso}(\mathbb{U})$-invariant Borel grey subset $A$ of $\operatorname{Mod}(\mathcal{L}, \mathbb{U})$ there exists a sentence $\phi$ of $\mathcal{L}_{\omega_{1} \omega}$ such that for all $M \in \operatorname{Mod}(\mathcal{L}, \mathbb{U})$ we have $A(M)=\phi^{M}$.

As an immediate consequence of Theorem 1.1, we obtain a López-Escobar theorem for

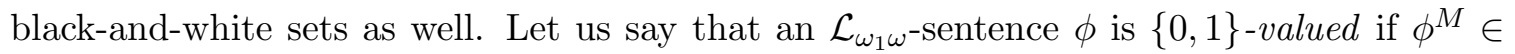
$\{0,1\}$ for every $M \in \operatorname{Mod}(\mathcal{L}, \mathbb{U})$. For such sentences $\phi$ we define $\operatorname{Mod}(\mathcal{L}, \mathbb{U}, \phi)$ to be the set of $M \in \operatorname{Mod}(\mathcal{L}, \mathbb{U})$ such that $\phi^{M}=0$.

Corollary 1.2. For every Iso(U)-invariant Borel subset $A$ of $\operatorname{Mod}(\mathcal{L}, \mathbb{U})$ there exists a sentence $\phi$ of $\mathcal{L}_{\omega_{1} \omega}$ such that $\phi$ is $\{0,1\}$-valued and $A=\operatorname{Mod}(\mathcal{L}, \mathbb{U}, \phi)$.

It is natural to ask whether the results hold with the Urysohn sphere replaced by another space $Y$. We remark that our proof applies if $Y$ is any approximately ultrahomogeneous, complete, separable metric space with a dense sequence $p_{n}$ satisfying the property: For every $n$, the Iso $(Y)$-orbit of $\left(p_{0}, \ldots, p_{n-1}\right)$ is definable in $Y^{n}$ in the sense of [BYBHU, Definition 9.16]. To see this, note that one can use [BYBHU, Proposition 9.19] to prove a suitable modification of Lemma 3.3.

After the first version of this paper had been posted on the Arxiv, a López-Escobar theorem for metric structures structures was also announced by Ben Yaacov, Nies, and Tsankov; see [BYNT]. While we work in the parametrization of $\mathcal{L}$-structures supported on $\mathbb{U}$ considered in [IMI], and for which the question of Ivanov and Majcher-Iwanow was formulated, the authors of [BYNT] consider a different parametrization of arbitrary separable $\mathcal{L}$-structures with a distinguished countable dense subset, which are coded by the sequences of values of all the predicates on such a subset. 
This article is organized as follows. In Section 2 we present several consequences of Theorem 1.1 and Corollary 1.2. For example, we show that the topological Vaught conjecture is equivalent to the natural formulation of the model-theoretic Vaught Conjecture in the context of model theory for metric structures. In Section 3 we introduce some technical components of the proof and state a theorem that is stronger than the main result. Finally, in Section 4 we prove this stronger theorem.

Acknowledgement. We would like to thank Itaï Ben Yaacov, Ilijas Farah, Bradd Hart, Luca Motto Ros, and Todor Tsankov for their comments and suggestions on earlier drafts of this paper.

\section{§2. Consequences of the main Result}

In this section we show that several standard applications of López-Escobar's theorem can be generalized to the setting of logic and model theory for metric structures.

Our first corollary is the existence of a Scott sentence that axiomatizes a single isomorphism class of structures (see for instance [G, Theorem 12.1.8], or [S, Theorem 4.2] for a metric version). Since the orbits of a Polish group action are always Borel (see [G, Proposition 3.1.10]) the following result is an immediate consequence of Corollary 1.2.

Corollary 2.1. For every $\mathcal{L}$-structure $M$ in $\operatorname{Mod}(\mathcal{L}, \mathbb{U})$ there is a sentence $\phi$ of $\mathcal{L}_{\omega_{1} \omega}$ such that $\phi$ is $\{0,1\}$-valued, and for any $N \in \operatorname{Mod}(\mathcal{L}, \mathbb{U})$ we have:

$$
\phi^{N}=0 \Longleftrightarrow M \cong N
$$

Next, recall that in Section 1 we observed that if $\phi$ is an $\mathcal{L}_{\omega_{1} \omega}$-sentence then the evaluation function $E_{\phi}$ is an $\operatorname{Iso}(\mathbb{U})$-invariant Borel grey subset of $\operatorname{Mod}(\mathcal{L}, \mathbb{U})$. In particular the $\operatorname{subspace} \operatorname{Mod}(\mathcal{L}, \mathbb{U}, \phi)$ of $\operatorname{Mod}(\mathcal{L}, \mathbb{U})$ consisting of just those $M$ with $\phi^{M}=0$ is a standard Borel Iso(U)-space. The next theorem will say that any standard Borel Iso(U)-space is isomorphic to an Iso(U)-space of this form.

First recall that if $E, F$ are equivalence relations on standard Borel spaces $X, Y$, then $E$ is Borel reducible to $F$ if there is a Borel function $f: X \rightarrow Y$ such that for $x, x^{\prime} \in X$,

$$
x E x^{\prime} \Longleftrightarrow f(x) F f\left(x^{\prime}\right) .
$$

If moreover such an $f$ can be taken to be a Borel isomorphism from $X$ to $Y$, then the equivalence relations $E$ and $F$ are said to be Borel isomorphic.

The following result implies that every orbit equivalence relation of a Polish group action is Borel isomorphic to the isomorphism relation on some $\operatorname{Mod}(\mathcal{L}, \mathbb{U}, \phi)$. In the statement, we say that a functional or relational symbol is 1-Lipschitz if its modulus of continuity is (bounded above by) the function $f(t)=t$.

Theorem 2.2. Let $\mathcal{L}$ be a relational countable language for continuous logic containing 1Lipschitz symbols of unbounded arity. Suppose that $G$ is a Polish group. If $X$ is a standard 
Borel $G$-space then there exists an $\mathcal{L}_{\omega_{1} \omega}$-sentence $\phi$, a continuous group monomorphism $\Phi: G \rightarrow \operatorname{Iso}(\mathbb{U})$, and a Borel injection $f: X \rightarrow \operatorname{Mod}(\mathcal{L}, \mathbb{U})$ such that:

$\circ \phi$ is $\{0,1\}$-valued;

$\circ \operatorname{rng}(f)=\operatorname{Mod}(\mathcal{L}, \mathbb{U}, \phi)$;

- $f$ maps distinct $G$-orbits into distinct Iso(U)-orbits; and

$\circ f$ is $\Phi$-equivariant, that is, for all $x \in X$ and $g \in G$ we have $f(g x)=\Phi(g) f(x)$.

Proof. Arguing as in the proof of [BK, Theorem 2.7.1(a)] we can assume without loss of generality that $\mathcal{L}$ is the language containing, for every $n \in \omega$, infinitely many 1-Lipschitz symbols $\left(R_{i}^{n}\right)_{i \in \omega}$ of arity $n$. (This can be done by replacing some 1-Lipschitz symbols with 1-Lipschitz symbols of higher arity that do not depend on the extra coordinates.)

We now claim that we can suppose without loss of generality that $G=\operatorname{Iso}(\mathbb{U})$ and $X=F(G)^{\omega}$. Here, $F(G)$ denotes the space of closed subsets of $G$ endowed with the Effros Borel structure [K, Section 12.C], and $G$ acts coordinatewise on $X$ by the left-shift. This claim follows from the following well-known facts:

o (Uspenskij [U1, U2]) $G$ is isomorphic to a closed subgroup of Iso(U).

○ (Mackey-Hjorth [G, Theorem 3.5.2]) If $G$ is a closed subgroup of the Polish group $H$ then every Polish $G$-space $X$ can be extended to a Polish $H$-space $\widetilde{X}$ in such a way that every $H$-orbit of $\widetilde{X}$ contains exactly one $G$-orbit of $X$.

- (Becker-Kechris [G, Theorem 3.3.4]) If $X$ is a Polish $G$-space then there is an equivariant embedding from $X$ into $F(G)^{\omega}$.

Next note that we can regard $G=\operatorname{Iso}(\mathbb{U})$ as a subspace of $\mathbb{U}^{\omega}$ by fixing a countable dense subset $\left(d_{n}\right)_{n \in \omega}$ in $\mathbb{U}$ and identifying each $g$ with the sequence $\left(g\left(d_{n}\right)\right)_{n \in \omega}$. Then it is easy to check that the map that sends a closed subset $F \subset \operatorname{Iso}(\mathbb{U})$ to its closure $\bar{F} \subset \mathbb{U}^{\omega}$ is a Borel embedding of Iso(U)-spaces. Hence we can suppose without loss of generality that $X=F\left(\mathbb{U}^{\omega}\right)^{\omega}$. For each sequence $F=\left(F_{i}\right)_{i \in \omega} \in X$ we will construct an element $M_{F} \in \operatorname{Mod}(\mathcal{L}, \mathbb{U})$ that codes $\left(F_{i}\right)_{i \in \omega}$ as follows. First for each $i$ we define a sequence of sets $A_{i}^{n} \subset \mathbb{U}^{n}$ by

$$
A_{i}^{n}=\left\{\bar{y} \in \mathbb{U}^{n} \mid \text { for every nbd } W \text { of } \bar{y} \text { we have }\left(W \times \mathbb{U}^{\omega}\right) \cap F_{i} \neq \emptyset\right\}
$$

It is easy to see the sets $A_{i}^{n}$ are closed. Moreover for each $i$ the sets $A_{i}^{n}$ form the levels of a tree which codes $F_{i}$ in the sense that $x \in F_{i}$ iff for all $n$ we have $x_{\mid n} \in A_{i}^{n}$. Now we define the structure $M_{F}$ by interpreting the symbol $R_{i}^{n}$ as the function

$$
\left(R_{i}^{n}\right)^{M_{F}}(\bar{y})=d\left(\bar{y}, A_{i}^{n}\right) .
$$

It is now straightforward to verify, as in the proof of [G, Theorem 3.6.1], that the function $f: F \mapsto M_{F}$ is a Borel embedding of $\operatorname{Iso}(\mathbb{U})$-spaces from $X$ to $\operatorname{Mod}(\mathcal{L}, \mathbb{U})$. By $[\mathrm{K}$, Corollary 15.2] the range of $f$ is a Borel subset of $\operatorname{Mod}(\mathcal{L}, \mathbb{U})$. It therefore follows from Corollary 1.2 that there is an $\mathcal{L}_{\omega_{1} \omega}$-sentence $\phi$ with the desired properties. 
A similar construction has been carried out with different methods in [IMI, Proposition 1.3]. Theorem 2.2 gives further confirmation for the intuition that $\mathbb{U}$ and Iso(U) play the same roles in model theory for metric structures as $\omega$ and $S_{\infty}$ play in first order model theory (for more examples see for instance the main results of $\left[\mathrm{EFP}^{+}\right]$and [IMI]).

We now give an application of Theorem 2.2 to the topological Vaught conjecture, which is the assertion that for every Polish group $G$ and standard Borel $G$-space $X$, either $X$ has just countably many orbits or it has perfectly many orbits (see [BK, Section 6.2]). Here, $X$ is said to have perfectly many orbits if there is an injective Borel reduction from the equality relation of $\mathbb{R}$ to the orbit equivalence relation of $G$ on $X$. In the following result, the implication $(1) \Rightarrow(2)$ is obvious, and $(2) \Rightarrow(1)$ is an immediate consequence of Theorem 2.2.

Corollary 2.3. Let $\mathcal{L}$ denote a relational countable language for continuous logic containing 1-Lipschitz symbols of unbounded arity. The following statements are equivalent:

(1) The topological Vaught conjecture holds;

(2) If $\phi$ is any $\mathcal{L}_{\omega_{1} \omega}$-sentence then $\operatorname{Mod}(\mathcal{L}, \mathbb{U}, \phi)$ has either countably many or perfectly many isomorphism classes.

Finally, we consider an application to infinitary logic. In López-Escobar's original work, he was interested foremost in establishing an interpolation property for the logic $\mathcal{L}_{\omega_{1} \omega}$. What we have called López-Escobar's theorem is in fact equivalent to this interpolation result. We will now show that a similar phenomenon holds in the setting of logic for metric structures. First we need an analog of the Luzin separation theorem [G, Theorem 1.6.1] for grey sets.

Proposition 2.4. (1) Let $X$ be a Polish space, and suppose that $A, B$ are grey subsets of $X, A$ is analytic, $B$ is coanalytic, and $A \geq B$. Then there is a Borel grey subset $C \sqsubseteq X$ such that $A \geq C \geq B$.

(2) Let $X$ be a Polish $G$-space, $A, B$ as above, and suppose additionally that $A, B$ are $G$-invariant. Then there is a $G$-invariant Borel grey subset $C \sqsubseteq X$ such that $A \geq$ $C \geq B$.

Proof. (a) Fix $r \in \mathbb{Q} \cap[0,1]$ and observe that $A_{<r} \subset B_{\leq r}$, where $A_{<r}$ is analytic and $B_{\leq r}$ is coanalytic. Therefore by [G, Theorem 1.6.1] there is $P^{(r)} \subset X$ Borel such that $A_{<r} \subset P^{(r)} \subset B_{\leq r}$. Now let $C \sqsubset X$ be the grey subset defined by

$$
C(x)=\inf \left\{r \in \mathbb{Q} \cap[0,1] \mid x \in P^{(r)}\right\} .
$$

It is straightforward to verify that $C$ is Borel and $A \geq C \geq B$.

(b) By part (a) there is a Borel grey subset $D$ of $X$ such that $A \geq D \geq B$. Define the grey subset $C$ of $X$ by $C(x) \leq r$ if and only if $\forall^{*} g \in G, D(g x) \leq r$. It is not difficult to verify by induction on the Borel rank of $D$ that $C$ is a Borel $G$-invariant subset of $X$ (see also Proposition 3.1). It is clear that $A \geq C \geq B$, which concludes the proof. 
We are now ready to prove the interpolation theorem for $\mathcal{L}_{\omega_{1} \omega}$. In the following if $\mathcal{L}$ and $\mathcal{R}$ are possibly distinct languages, and $\phi$ is an $\mathcal{L}_{\omega_{1} \omega^{-}}$-sentence, and $\rho$ is an $\mathcal{R}_{\omega_{1} \omega^{-}}$-sentence, then we write $\phi \models \rho$ iff $\phi^{M} \geq \rho^{M}$ for every $M \in \operatorname{Mod}(\mathcal{L} \cup \mathcal{R}, \mathbb{U})$.

Corollary 2.5. The logic $\mathcal{L}_{\omega_{1} \omega}$ has the following interpolation property: Suppose that $\mathcal{L}, \mathcal{R}, \mathcal{S}$ are pairwise disjoint countable languages, $\phi$ is a sentence in $(\mathcal{L} \cup \mathcal{R})_{\omega_{1} \omega}$ and $\rho$ is a sentence in $(\mathcal{L} \cup \mathcal{S})_{\omega_{1} \omega}$. If $\phi \models \rho$, then there is an $\mathcal{L}_{\omega_{1} \omega}$-sentence $\tau$ such that $\phi \models \tau$ and $\tau \models \rho$.

Proof. We can canonically identify $\operatorname{Mod}(\mathcal{L} \cup \mathcal{S}, \mathbb{U})$ with $\operatorname{Mod}(\mathcal{L}, \mathbb{U}) \times \operatorname{Mod}(\mathcal{S}, \mathbb{U})$ and $\operatorname{Mod}(\mathcal{L} \cup$ $\mathcal{R}, \mathbb{U})$ with $\operatorname{Mod}(\mathcal{L}, \mathbb{U}) \times \operatorname{Mod}(\mathcal{R}, \mathbb{U})$. Define $A$ to be the analytic subset of $\operatorname{Mod}(\mathcal{L}, \mathbb{U})$

$$
A=\inf _{M \in \operatorname{Mod}(\mathcal{S}, \mathbb{U})} E_{\phi}
$$

where $E_{\phi} \sqsubseteq \operatorname{Mod}(\mathcal{L}, \mathbb{U}) \times \operatorname{Mod}(\mathcal{S}, \mathbb{U})$. Similarly define $B$ to be the coanalytic subset of $\operatorname{Mod}(\mathcal{L}, \mathbb{U})$

$$
B=\sup _{M \in \operatorname{Mod}(\mathcal{S}, \mathbb{U})} E_{\rho} .
$$

Observe that $A \geq B$ since $\phi \models \rho$. Therefore by Proposition 2.4 there is a Iso(U)-invariant Borel grey subset $C$ of $\operatorname{Mod}(\mathcal{L}, \mathbb{U})$ such that $A \geq C \geq B$. By Theorem 1.1 there is an

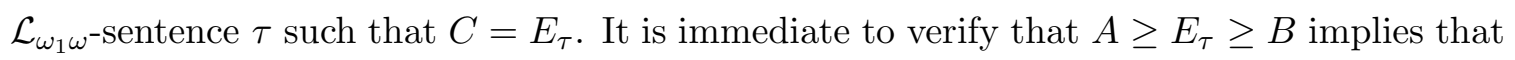
$\phi \models \tau$ and $\tau \models \rho$.

\section{§3. Further notions And A STREnGthening of the MAin RESUlt}

In this section we formulate a statement that is stronger than Theorem 1.1 and handles the case when $A$ is a grey subset that is not invariant. Our motivation for this proof strategy comes from Vaught's dynamical proof of López-Escobar's theorem (see [V] or [K, Theorem $7.8])$.

In order to state the stronger result, we will need to introduce the following category quantifiers for grey sets. These generalize the classical category quantifiers $\exists^{*}$ and $\forall^{*}$ as defined for instance in [K, Section 8.J]. If $X, Y$ are Polish spaces, $U \subset Y$ is open, and $B$ is a grey subset of $X \times Y$, then we define the grey subsets inf ${ }_{y \in U}^{*} B$ and $\sup _{y \in U}^{*} B$ of $X$ by the properties:

$$
\begin{aligned}
\left(\inf _{y \in U}^{*} B\right)(x)<r & \exists^{*} y \in U \text { such that } B(x, y)<r \\
\left.\sup _{y \in U}^{*} B\right)(x)>r & \Longleftrightarrow \exists^{*} y \in U \text { such that } B(x, y)>r .
\end{aligned}
$$

The next proposition lists some of the basic properties of these set-theoretic category quantifiers. They can be proved with the same arguments as Propositions 3.2.5, 3.2.6, and Theorem 3.2.7 of [G] (in [G, 3.2.5-3.2.7] the space $Y$ of Proposition 3.1 appears as a Polish group $G$ acting on $X$ ). Note that in the statement, as in the rest of this article, all the usual arithmetic operations in fact denote their truncated versions to the interval $[0,1]$. For 
example if $a, b \in \mathbb{R}$ then $a+b$ stands for

$$
\max \{0, \min \{1, a+b\}\}
$$

and similarly for the other operations.

Proposition 3.1. Let $X, Y$ be Polish, $U \subset Y$ open, and $B$ a grey subset of $X \times Y$.

(1) $\inf _{y \in U}^{*}(q-B)=q-\sup _{y \in U}^{*} B$ for any $q \in[0,1]$;

(2) $\sup _{y \in U}^{*} B=\sup _{n \in \omega} \inf _{y \in W_{n} \cap U}^{*} B$, where $W_{n}$ enumerates a basis for $Y$;

(3) If $B_{n}$ is a sequence of grey subsets of $X \times Y$, then $\inf _{n} \inf _{y}^{*} B_{n}=\inf _{y}^{*} \inf _{n} B_{n}$ and $\sup _{y \in U}^{*} \sup _{n} B_{n}=\sup _{n} \sup _{y \in U}^{*} B_{n}$

(4) If $B$ is open then $\inf _{y \in U}^{*} B$ is open;

(5) If $B$ is $\boldsymbol{\Sigma}_{\alpha}^{0}$ then $\inf _{y \in U}^{*} B$ is $\boldsymbol{\Sigma}_{\alpha}^{0}$;

(6) If $B$ is $\boldsymbol{\Pi}_{\alpha}^{0}$ then $\sup _{y \in U}^{*} B$ is $\boldsymbol{\Pi}_{\alpha}^{0}$.

Although we will refrain from using the notation in our proof, it is worth remarking that the category quantifiers can be used to define a version of the Vaught transforms in the grey setting. (The grey Vaught transforms were first introduced in [IMI, Section 2.1].) If $X$ is a Polish $G$-space, $A \sqsubseteq X$ is Borel, and $U \sqsubseteq G$ is open, then

$$
\begin{aligned}
A^{* U}(x) & =\sup _{g \in G}^{*}(A(g x)-U(g)), \text { and } \\
A^{\triangle U}(x) & =\inf _{g \in G}^{*}(A(g x)+U(g)) .
\end{aligned}
$$

The basic properties of the Vaught transforms listed in [IMI, Lemma 2.4] can easily be obtained as a consequence of Proposition 3.1.

We will also need some notation for a family of "basic" open graded subsets of Iso(U). We fix once and for all an enumeration $\mathbf{p}=\left(p_{n}\right)_{n \in \omega}$ of a dense subset of $\mathbb{U}$. For any $u \in \mathbb{U}^{k}$ we define the open grey subset $[u]$ of Iso(U) by

$$
[u](g)=d\left(g^{-1} \mathbf{p}_{\mid k}, u\right) .
$$

(Here as usual $d$ denotes the maximum metric on $\mathbb{U}^{k}$.) We also let $\mathcal{O}\left(\mathbf{p}_{\mid k}\right)$ be the orbit of $\mathbf{p}_{\mid k}$ under the action of Iso(U), which coincides with the set of realizations of the type of the $k$-tuple $\mathbf{p}_{\mid k}$. The level sets $[u]_{<r}$, where $u \in \mathcal{O}\left(\mathbf{p}_{\mid k}\right)$ and $r>0$, form an open basis for the topology of Iso(U).

We are now ready to state our strengthening of Theorem 1.1. Roughly speaking, the result accommodates Borel graded sets that are not invariant, at the cost of taking a Vaught transform and allowing parameters in the formula $\phi$. In the statement, we say that a formula $\phi$ is $N$-Lipschitz if its modulus of continuity is bounded above by the function $f(t)=N t$.

Theorem 3.2. Suppose that $\mathcal{L}$ is a countable language for continuous logic, $\mathbf{p}$ is as above, and $k \in \mathbb{N}$. For any Borel grey subset $A \sqsubseteq \operatorname{Mod}(\mathcal{L}, \mathbb{U})$ and for any $N \in \mathbb{N}$ there exists an $N$-Lipschitz $\mathcal{L}_{\omega_{1} \omega}$-formula $\phi$ with $k$ free variables such that for every $M \in \operatorname{Mod}(\mathcal{L}, \mathbb{U})$ and 
$u \in \mathbb{U}^{k}$, we have

$$
\sup _{g \in \operatorname{Iso}(\mathbb{U})}^{*}\left[A(g M)-N d\left(g^{-1} \mathbf{p}_{\mid k}, u\right)\right]=\phi^{M}(u) .
$$

Theorem 1.1 follows as the special case when $k=0$ and $N=1$. Indeed, if $A$ is an Iso(U)-invariant grey subset of $\operatorname{Mod}(\mathcal{L}, \mathbb{U})$, then

$$
A(M)=\sup _{g \in \operatorname{Iso}(\mathbb{U})}^{*} A(g M) .
$$

Therefore Theorem 3.2 yields a sentence $\phi$ such that $A(M)=\phi^{M}$ for $M \in \operatorname{Mod}(\mathcal{L}, \mathbb{U})$.

In the proof of the theorem we will need the following perturbation result, which is similar to [IMI, Lemma 2.3]. In the statement, we denote by $\tau_{k}(\bar{x}, \bar{y})$ the quantifier-free formula with $2 k$ free variables given by

$$
\max _{i, j \in k}\left|d\left(x_{i}, x_{j}\right)-d\left(y_{i}, y_{j}\right)\right| .
$$

Observe that $\tau\left(\bar{x}, \mathbf{p}_{\mid k}\right)$ can be regarded as a quantifier-free formula with $k$ variables.

Lemma 3.3. For all $\varepsilon>0$, if $u, w \in \mathbb{U}^{k}$ are such that $\tau_{k}(u, w)<\varepsilon$, then there is $g \in \operatorname{Iso}(Y)$ such that $d(u, g w)<3 \varepsilon$.

Proof. Consider the metric space $Z$ obtained from the disjoint union of $\left\{u_{i}: i \in k\right\}$ and $\left\{w_{i}: i \in k\right\}$ as in $[\mathrm{P}$, Example 56], where

$$
d\left(u_{i}, w_{j}\right)=\min _{n \in k}\left(d\left(u_{i}, u_{n}\right)+\varepsilon+d\left(w_{n}, w_{j}\right)\right) .
$$

By the finite injectivity of Urysohn space [M2] the isometric embedding of $\left\{u_{i}: i \in k\right\}$ in $\mathbb{U}$ extends to an isometric embedding of $Z$ into $\mathbb{U}$. This gives $\widetilde{w}=\left(\widetilde{w}_{j}\right)_{j \in k} \in \mathbb{U}^{k}$ such that

$$
\begin{gathered}
d(\widetilde{w}, u)<3 \varepsilon, \text { and } \\
d\left(\widetilde{w}_{i}, \widetilde{w}_{j}\right)=d\left(w_{i}, w_{j}\right)
\end{gathered}
$$

for $i, j \in k$. Since $\mathbb{U}$ is ultrahomogeneous, there is an isometry $g \in \operatorname{Iso}(\mathbb{U})$ such that $g w=\widetilde{w}$ and hence $d(g w, u)<3 \varepsilon$.

We remark that Lemma 3.3 together with [BYBHU, Proposition 9.19] implies that $\mathcal{O}\left(\mathbf{p}_{\mid k}\right)$ is a definable subset of $\mathbb{U}^{k}$ in the sense of [BYBHU, Definition 9.16].

\section{$\S 4$. THE PROOF}

In this section, we give the proof of Theorem 3.2. To begin, we let $\mathcal{B}$ denote the family of Borel grey subsets of $\operatorname{Mod}(\mathcal{L}, \mathbb{U})$ which satisfy the conclusion of Theorem 3.2 for all $k \in \mathbb{N}$. Our strategy will be to show that $\mathcal{B}$ has the following properties.

(1) If $A \in \mathcal{B}$ then $q-A \in \mathcal{B}$ for every $q \in[0,1]$ (Section 4.1);

(2) For every $n \in \mathbb{N}$ and every quantifier-free $\mathcal{L}_{\omega \omega}$-formula $\phi(\bar{x})$ with $n$ free variables the grey subset $E_{\phi, \mathbf{p}_{\mid n}}$ of $\operatorname{Mod}(\mathcal{L}, Y)$ defined by

$$
E_{\phi, \mathbf{p}_{\mid n}}(M)=\phi^{M}\left(\mathbf{p}_{\mid n}\right)
$$


is in $\mathcal{B}$ (Section 4.2);

(3) If $A, B \in \mathcal{B}$ and $\lambda, \mu \in[0,1]$ then $\lambda A+\mu B \in \mathcal{B}$ (Section 4.3);

(4) If $A_{n} \in \mathcal{B}$ for every $n \in \omega$, then $\inf _{n} A_{n} \in \mathcal{B}$ and $\sup _{n} A_{n} \in \mathcal{B}$ (Section 4.4).

We once again remind the reader that in (3), as everywhere, the arithmetic operations denote their truncated versions.

We now show that these facts ensure that the family $\mathcal{B}$ contains all Borel grey subsets of $\operatorname{Mod}(\mathcal{L}, \mathbb{U})$. For this we need the following lemma. In the statement, recall that a family of functions separates the points of $X$ if for every distinct $x, y \in X$ there is $f$ in the family such that $f(x) \neq f(y)$.

Lemma 4.1. Suppose that $X$ is a standard Borel space, $\mathcal{F}$ is a family of Borel grey sets of $X$, and $\mathcal{F}_{0} \subset \mathcal{F}$ is a countable subfamily that separates the points of $X$. Assume further that $\mathcal{F}$ satisfies the following closure properties:

(1) If $A \in \mathcal{F}$ then $q-A \in \mathcal{F}$ for every $q \in[0,1]$;

(2) Every constant function belongs to $\mathcal{F}$.

(3) If $A, B \in \mathcal{F}$ and $\lambda, \mu \in[0,1]$ then $\lambda A+\mu B \in \mathcal{F}$;

(4) If $A_{n} \in \mathcal{F}$ for every $n \in \omega$, then $\inf _{n} A_{n} \in \mathcal{F}$ and $\sup _{n} A_{n} \in \mathcal{F}$;

Then $\mathcal{F}$ contains all Borel grey sets.

Proof. By induction it follows from $(3)$ that $\mathcal{F}$ is closed under arbitrary finite linear combinations with coefficients in $[0,1]$. Moreover one can deduce from (4) that $\mathcal{F}$ is closed under pointwise limits. Arguing as in the proof of [K, Theorem 11.6] one can show that any Borel grey set is a pointwise limit of linear combinations of $\{0,1\}$-valued Borel grey sets. Therefore it is enough to show that for every Borel subset $U$ of $X$, the zero-indicator $\mathbf{0}_{U}$ of $U$ lies in $\mathcal{F}$. (Here the zero indicator $\mathbf{0}_{U}$ is the function constantly equal to 0 on $U$ and constantly equal to 1 on $X \backslash U$; see [BYM, Notation 1.2].)

For this, let $\mathcal{U}$ denote the family of Borel subsets $U$ of $X$ such that $\mathbf{0}_{U} \in \mathcal{F}$. Also let $\mathcal{U}_{0}$ denote the family of level sets $A_{\leq q}$ for $A \in \mathcal{F}_{0}$ and $q \in \mathbb{Q} \cap[0,1]$. It follows from (1) and (4) that $\mathcal{U}$ is a $\sigma$-algebra of Borel subsets of $X$. Moreover since $\mathcal{F}_{0}$ separates the points of $X$, $\mathcal{U}_{0}$ is a countable family of Borel sets that separate the points of $X$. By [M1, Theorem 3.3] in order to show that $\mathcal{U}$ contains all Borel sets it is enough to prove that $\mathcal{U}_{0}$ is contained in $\mathcal{U}$. For this, observe that for each $A \in \mathcal{F}_{0}$ and $q \in \mathbb{Q} \cap[0,1]$ the indicator function $\mathbf{0}_{A_{\leq q}}$ of the level set $A_{\leq q}$ is $\sup _{m \in \mathbb{N}} m(A-q)$. By (2), and (3) we have $m(A-q) \in \mathcal{F}$ for every $m \in \mathbb{N}$ and hence $\mathbf{0}_{A_{\leq q}} \in \mathcal{F}$ by (4). Therefore $A_{\leq q} \in \mathcal{U}$, as claimed.

We may now give the conclusion of the proof of the main theorem.

Proof of Theorem 3.2. By Lemmas 4.4, 4.7, 4.9, and 4.10 below, the family $\mathcal{B}$ of grey sets satisfying the conclusion of the theorem satisfies hypotheses (1), (2), (3), (4) of Lemma 4.1. 
Let $\mathcal{B}_{0}$ denote the family of grey subsets of $\operatorname{Mod}(\mathcal{L}, \mathbb{U})$ of the form

$$
\begin{aligned}
& M \mapsto R^{M}\left(p_{i_{0}}, \ldots, p_{i_{n-1}}\right), \text { or } \\
& M \mapsto d\left(f^{M}\left(p_{i_{0}}, \ldots, p_{i_{n-1}}\right), p_{i_{n}}\right)
\end{aligned}
$$

where $i_{0}, \ldots, i_{n} \in \mathbb{N}$ and $f, R$ are $n$-ary symbols of $\mathcal{L}$. It is straightforward to verify that $\mathcal{B}_{0}$ separates the points of $\operatorname{Mod}(\mathcal{L}, \mathbb{U})$. Moreover, by Lemma $4.7, \mathcal{B}_{0}$ is contained in $\mathcal{B}$. It therefore follows from Lemma 4.1 that $\mathcal{B}$ contains all Borel grey sets, as desired.

We now proceed to verify each of the closure properties outlined at the beginning of this section.

§4.1. Negation. Recall that $\mathcal{O}\left(\mathbf{p}_{\mid k}\right)$ for $k \in \omega$ denotes the orbit of $\mathbf{p}_{\mid k}$ under the action $\operatorname{Iso}(\mathbb{U}) \curvearrowright \mathbb{U}^{k}$.

Lemma 4.2. Suppose that $A$ is a grey subset of $\operatorname{Mod}(\mathcal{L}, \mathbb{U}), k, N \in \omega$ with $N \geq 1$, and $u \in \mathbb{U}^{k}$. For any $t \in[0,1]$, the following statements are equivalent:

(1) $\inf _{g \in \operatorname{Iso}(\mathbb{U})}^{*}\left[A(g M)+N d\left(u, g^{-1} \mathbf{p}_{\mid k}\right)\right]<t$;

(2) there are $\widetilde{k} \geq k, \widetilde{N} \geq N$, and $\widetilde{u} \in \mathcal{O}\left(\mathbf{p}_{\mid \widetilde{k}}\right)$ such that

$$
N d\left(\widetilde{u}_{\mid k}, u\right)+\sup _{g \in \operatorname{Iso}(\mathbb{U})}^{*}\left[A(g M)-\widetilde{N} d\left(\widetilde{u}, g^{-1} \mathbf{p}_{\mid \widetilde{k}}\right)\right]<t
$$

(3) there are $t_{0}<t, \widetilde{k} \geq k, \widetilde{N} \geq N$, such that for every $m \geq 1$ there is $\widetilde{u} \in \mathbb{U}^{\widetilde{k}}$ such that

$$
m \tau_{\widetilde{k}}\left(\widetilde{u}, \mathbf{p}_{\mid \widetilde{k}}\right)+N d\left(\widetilde{u}_{\mid k}, u\right)+\sup _{g \in \operatorname{Iso}(\mathbb{U})}^{*}\left[A(g M)-\widetilde{N} d\left(\widetilde{u}, g^{-1} \mathbf{p}_{\mid \widetilde{k}}\right)\right]<t_{0} .
$$

Proof. (1) $\Rightarrow(2)$ Suppose that

$$
\inf _{g \in \operatorname{Iso}(\mathbb{U})}^{*}\left[A(g M)+N d\left(u, g^{-1} \mathbf{p}_{\mid k}\right)\right]<t .
$$

Thus there are $s, r \in[0,1]$ such that $s+r<t$ and $\exists^{*} g \in \operatorname{Iso}(\mathbb{U})$ such that $A(g M)<r$ and $N d\left(u, g^{-1} \mathbf{p}_{\mid k}\right)<s$. In particular there is a nonempty open $U \subset[u]_{<s N^{-1}}$ such that $\forall^{*} g \in U, A(g M)<r$. Pick $g_{0} \in U$ and observe that $N d\left(u, g_{0}^{-1} \mathbf{p}_{\mid k}\right)<s$. Define $\widetilde{k} \geq k$ and $\widetilde{N} \geq N$ such that if $g \in \operatorname{Iso}(\mathbb{U})$ is such that $\widetilde{N} d\left(g^{-1} \mathbf{p}_{\mid \widetilde{k}}, g_{0}^{-1} \mathbf{p}_{\mid \widetilde{k}}\right)<1$ then $g \in U$. Define $\widetilde{u}=g_{0}^{-1} \mathbf{p}_{\mid \widetilde{k}} \in \mathcal{O}\left(\mathbf{p}_{\mid \widetilde{k}}\right)$. Observe that

$$
N d\left(\widetilde{u}_{\mid k}, u\right)=N d\left(g_{0}^{-1} \mathbf{p}_{\mid k}, u\right)<s .
$$

Moreover $\forall^{*} g \in[\widetilde{u}]_{<\widetilde{N}^{-1}}$,

$$
A(g M)<r \leq r+\widetilde{N} d\left(\widetilde{u}, g^{-1} \mathbf{p}_{\mid \widetilde{k}}\right)
$$

Therefore

$$
\sup _{g \in \operatorname{Iso}(\mathbb{U})}^{*}\left[A(g M)-\widetilde{N} d\left(\widetilde{u}, g^{-1} \mathbf{p}_{\mid \widetilde{k}}\right)\right] \leq r
$$

and hence

$$
N d\left(\widetilde{u}_{\mid k}, u\right)+\sup _{g \in \operatorname{Iso}(\mathbb{U})}^{*}\left[A(g M)-\widetilde{N} d\left(\widetilde{u}, g^{-1} \mathbf{p}_{\mid \widetilde{k}}\right)\right] \leq r+s<t
$$


$(2) \Rightarrow(3)$ This is obvious, since $\widetilde{u} \in \mathcal{O}\left(\mathbf{p}_{\mid k}\right)$ implies $\tau_{\widetilde{k}}\left(\widetilde{u}, \mathbf{p}_{\mid \widetilde{k}}\right)=0$.

$(3) \Rightarrow(2)$ By hypothesis there are $t_{0}<t, \widetilde{k} \geq k, \widetilde{N} \geq N$, such that for every $m \geq 1$ there is $\widetilde{u} \in \mathbb{U}^{\widetilde{k}}$ such that

$$
m \tau_{\widetilde{k}}\left(\widetilde{u}, \mathbf{p}_{\mid \widetilde{k}}\right)+N d\left(\widetilde{u}_{\mid k}, u\right)+\sup _{g \in \operatorname{Iso}(\mathbb{U})}^{*}\left[A(g M)-\widetilde{N} d\left(\widetilde{u}, g^{-1} \mathbf{p}_{\mid \widetilde{k}}\right)\right]<t_{0} .
$$

Fix $m \in \mathbb{N}$ such that $t_{0}+\frac{6 \widetilde{N}}{m}<t$. Let $\widetilde{u} \in \mathbb{U}^{\widetilde{k}}$ be such that

$$
m \tau_{\widetilde{k}}\left(\widetilde{u}, \mathbf{p}_{\mid \widetilde{k}}\right)+N d\left(\widetilde{u}_{\mid k}, u\right)+\sup _{g \in \operatorname{Iso}(\mathbb{U})}^{*}\left[A(g M)-\widetilde{N} d\left(\widetilde{u}, g^{-1} \mathbf{p}_{\mid \widetilde{k}}\right)\right]<t_{0} .
$$

Since $\tau_{\widetilde{k}}\left(\widetilde{u}, \mathbf{p}_{\mid \widetilde{k}}\right)<1 / m$, by Lemma 3.3 there is $g_{0} \in \operatorname{Iso}(\mathbb{U})$ such that $d(\widetilde{v}, \widetilde{u})<3 / m$, where $\widetilde{v}=g_{0}^{-1} \mathbf{p}_{\mid \widetilde{k}} \in \mathcal{O}\left(\mathbf{p}_{\mid \widetilde{k}}\right)$. Observe now that

$$
\begin{aligned}
& N d\left(\widetilde{v}_{\mid k}, u\right)+\sup _{g \in \operatorname{Iso}(\mathbb{U})}^{*}\left[A(g M)-\widetilde{N} d\left(\widetilde{v}, g^{-1} \mathbf{p}_{\mid \widetilde{k}}\right)\right] \\
\leq & N d\left(\widetilde{u}_{\mid k}, u\right)+\sup _{g \in \operatorname{Iso}(\mathbb{U})}\left[A(g M)-\widetilde{N} d\left(\widetilde{u}, g^{-1} \mathbf{p}_{\mid \widetilde{k}}\right)\right]+2 \widetilde{N} d(\widetilde{v}, \widetilde{u}) \\
\leq & t_{0}+\frac{6 \widetilde{N}}{m}<t .
\end{aligned}
$$

$(2) \Rightarrow(1)$ By hypothesis there are $\widetilde{k} \geq k, \widetilde{N} \geq N$, and $\widetilde{u} \in \mathcal{O}\left(\mathbf{p}_{\mid \widetilde{k}}\right)$ such that

$$
N d\left(\widetilde{u}_{\mid k}, u\right)+\sup _{g \in \operatorname{Iso}(\mathbb{U})}^{*}\left[A(g M)-\tilde{N} d\left(\widetilde{u}, g^{-1} \mathbf{p}_{\mid \widetilde{k}}\right)\right]<t .
$$

Define $s=N d\left(\widetilde{u}_{\mid k}, u\right)$ and

$$
r=\sup _{g \in \operatorname{Iso}(\mathbb{U})}^{*}\left[A(g M)-\widetilde{N} d\left(\widetilde{u}, g^{-1} \mathbf{p}_{\mid \widetilde{k}}\right)\right] .
$$

Fix $\delta>0$ such that $s+r+2 \delta<t$. Observe that since $\widetilde{u} \in \mathcal{O}\left(\mathbf{p}_{\tilde{k}}\right)$ we have that $[\widetilde{u}]_{<\delta \widetilde{N}^{-1}} \neq$ $\varnothing$. Moreover $[\widetilde{u}]_{<\delta \widetilde{N}^{-1}} \subset[u]_{<(s+\delta) N^{-1}}$. In fact suppose that $g \in[\widetilde{u}]_{<\delta \widetilde{N}^{-1}}$ and hence $\widetilde{N} d\left(g^{-1} \mathbf{p}_{\mid \widetilde{k}}, \widetilde{u}\right)<\delta$. Thus we have

$$
\begin{aligned}
N d\left(g^{-1} \mathbf{p}_{\mid k}, u\right) & \leq N d\left(\widetilde{u}_{\mid k}, u\right)+N d\left(\widetilde{u}_{\mid k}, g^{-1} \mathbf{p}_{\mid k}\right) \\
& \leq s+\widetilde{N} d\left(\widetilde{u}, g^{-1} \mathbf{p}_{\mid \widetilde{k}}\right) \\
& \leq s+\delta .
\end{aligned}
$$

Moreover have that $\forall^{*} g \in[\widetilde{u}]_{<\delta \widetilde{N}^{-1}}$,

$$
A(g M) \leq r+\widetilde{N} d\left(\widetilde{u}, g^{-1} \mathbf{p}_{\mid \widetilde{k}}\right)<r+\delta .
$$

It follows that $[u]_{<(s+\delta) N^{-1}} \neq \varnothing$ and $\exists^{*} g \in[u]_{<(s+\delta) N^{-1}}$ such that $A(g M)<r+\delta$. Therefore

$$
\inf _{g \in \operatorname{Iso}(\mathbb{U})}^{*}\left[A(g M)+N d\left(u, g^{-1} \mathbf{p}_{\mid k}\right)\right] \leq s+\delta+r+\delta<t .
$$

This concludes the proof.

Lemma 4.3. If $A$ is a grey subset of $\operatorname{Mod}(\mathcal{L}, \mathbb{U})$, then $A \in \mathcal{B}$ if and only if for every $k, N \in \omega$ with $N \geq 1$ there is an $N$-Lipschitz formula $\varphi$ such that, for every $M \in \operatorname{Mod}(\mathcal{L}, \mathbb{U})$,

$$
\inf _{g \in \operatorname{Iso}(\mathbb{U})}^{*}\left[A(g M)+N d\left(g^{-1} \mathbf{p}_{\mid k}, u\right)\right]=\varphi^{M}(u) .
$$


Proof. Suppose that $A \in \mathcal{B}$. We have that for every $\widetilde{k}, \widetilde{N} \in \omega$ such that $\widetilde{N} \geq 1$ there is a formula $\psi_{\widetilde{k}, \widetilde{N}}$ in $\widetilde{k}$ free variables such that

$$
\sup _{g \in \operatorname{Iso}(\mathbb{U})}^{*}\left[A(g M)-\widetilde{N} d\left(u, g^{-1} \mathbf{p}_{\mid \widetilde{k}}\right)\right]=\psi_{\widetilde{k}, \widetilde{N}}^{M}(u) .
$$

Fix $k, N \in \omega$ with $N \geq 1$. Observe that for every $\widetilde{N}, m, k \in \omega$

$$
\inf _{y_{0}, \ldots, y_{\widetilde{k}-1}}\left[m \tau_{\widetilde{k}}\left(\bar{y}, \mathbf{p}_{\widetilde{k}}\right)+N d\left(\bar{y}_{\mid k}, \bar{x}\right)+\psi_{\widetilde{k}, \widetilde{N}}(\bar{y})\right]
$$

is an $N$-Lipschitz formula in the $k$ free variables $\bar{x}$. Therefore

$$
\inf _{\widetilde{N} \geq N} \inf _{\widetilde{k} \geq k} \sup _{m \geq 1} \inf _{y_{0}, \ldots, y_{\widetilde{k}-1}}\left[m \tau_{\widetilde{k}}\left(\bar{y}, \mathbf{p}_{\mid \widetilde{k}}\right)+N d\left(\bar{y}_{\mid k}, \bar{x}\right)+\psi_{\widetilde{k}, \widetilde{N}}(\bar{y})\right]
$$

is also an $N$-Lipschitz formula $\varphi(\bar{x})$ in the $k$ free variables $\bar{x}$. Moreover it follows from Lemma 4.2 that Equation (1) holds. Conversely suppose that for every $k, N \in \omega$ with $N \geq 1$ there exists and $N$-Lipschitz formula $\varphi$ such that Equation (1) holds. Performing the substitution $x \mapsto 1-x$ in each side of Equation (1) shows that $1-A \in \mathcal{B}$. By the proof above applied to $1-A$ we conclude that for every $k, N \in \omega$ with $N \geq 1$ there is an $N$-Lipschitz formula $\psi$ such that

$$
\inf _{g \in \operatorname{Iso}(\mathbb{U})}^{*}\left[(1-A)(g M)+N d\left(g^{-1} \mathbf{p}_{\mid k}, u\right)\right]=\psi^{M}(u) .
$$

Performing again the substitution $x \mapsto 1-x$ gives

$$
\sup _{g \in \operatorname{Iso}(\mathbb{U})}^{*}\left[A(g M)-N d\left(g^{-1} \mathbf{p}_{\mid k}, u\right)\right]=(1-\psi)^{M}(u) .
$$

Therefore the fomula $1-\psi$ witnesses the fact that $A \in \mathcal{B}$.

With a similar argument as in the proof of Lemma 4.3 one can prove the following lemma.

Lemma 4.4. Suppose that $A$ is a grey subset of $\operatorname{Mod}(\mathcal{L}, \mathbb{U})$ and $q \in[0,1]$. Then $A \in \mathcal{B}$ if and only if $q-A \in \mathcal{B}$.

§4.2. The base case. The proofs of Lemma 4.5 and Lemma 4.6 are analogous to the proof of Lemma 4.2, and are omitted. The key point is that one can perturb an element of $\mathbb{U}^{k}$ for which $\tau_{k}\left(w, \mathbf{p}_{\mid k}\right)$ is small to an element in the orbit $\mathcal{O}\left(\mathbf{p}_{\mid k}\right)$ of $\mathbf{p}_{\mid k}$ using Lemma 3.3.

Lemma 4.5. Suppose that $n, k \in \omega, \phi$ is a quantifier-free $\mathcal{L}_{\omega \omega}$-formula with $n$ free variables, and $u \in \mathbb{U}^{k}$. If $n<k$ and $t \in[0,1]$, then following statements are equivalent:

(1) $\inf _{g \in \text { Iso }(\mathbb{U})}^{*}\left[\phi^{M}\left(g^{-1} \mathbf{p}_{\mid n}\right)+N d\left(g^{-1} \mathbf{p}_{\mid k}, u\right)\right]<t$;

(2) There is $w \in \mathcal{O}\left(\mathbf{p}_{\mid k}\right)$ such that $\phi^{M}\left(w_{\mid n}\right)+N d(w, u)<t$;

(3) There is $t_{0}<t$ such that for every $m \geq 1, \exists w \in \mathbb{U}^{k}$ such that $\phi^{M}\left(w_{\mid n}\right)+N d(w, u)+$ $m \tau_{k}\left(w, \mathbf{p}_{\mid k}\right)<t_{0}$.

Lemma 4.6. Suppose that $n, k \in \omega, \phi$ is a quantifier-free $\mathcal{L}_{\omega \omega}$-formula with $n$ free variables, and $u \in \mathbb{U}^{k}$. If $k \leq n$ and $t \in[0,1]$, then the following statements are equivalent:

(1) $\inf _{g \in \operatorname{Iso}(\mathbb{U})}^{*}\left[\phi^{M}\left(g^{-1} \mathbf{p}_{\mid n}\right)+N d\left(g^{-1} \mathbf{p}_{\mid k}, u\right)\right]<t$; 
(2) There is $w \in \mathcal{O}\left(\mathbf{p}_{\mid n}\right)$ such that $\phi^{M}(w)+N d\left(w_{\mid k}, u\right)<t$;

(3) There is $t_{0}<t$ such that for every $m \geq 1$ there is $w \in \mathbb{U}^{n}$ such that $\phi^{M}(w)+$ $N d\left(w_{\mid k}, u\right)+m \tau_{n}\left(w, \mathbf{p}_{\mid n}\right)<t_{0}$.

Lemma 4.7. If $\phi$ is an $\mathcal{L}_{\omega \omega}$ quantifier-free formula with $n$ free variables, then the grey subset $M \mapsto \phi^{M}\left(g^{-1} \mathbf{p}_{\mid n}\right)$ of $\operatorname{Mod}(\mathcal{L}, \mathbb{U})$ belongs to $\mathcal{B}$.

Proof. By Lemma 4.3 it is enough to show that for every $N, k \in \omega$ with $N \geq 1$ there is an $N$-Lipschitz $\mathcal{L}_{\omega_{1} \omega}$ formula $\psi$ with $k$ free variables such that

$$
\inf _{g \in \operatorname{Iso}(\mathbb{U})}^{*}\left[\phi^{M}\left(g^{-1} \mathbf{p}_{\mid n}\right)+N d\left(g^{-1} \mathbf{p}_{\mid k}, u\right)\right]=\psi^{M}(u)
$$

for every $M \in \operatorname{Mod}(\mathcal{L}, \mathbb{U})$ and $u \in \mathbb{U}^{k}$. Let us distinguish the cases when $n<k$ and $n \geq k$. If $n<k$ define the $N$-Lipschitz formula $\psi(\bar{x})$ in the $k$ free variables $\bar{x}$ by

$$
\sup _{m \geq 1} \inf _{y_{0}, \ldots, y_{k-1}}\left[m \tau_{k}\left(\bar{y}, \mathbf{p}_{\mid k}\right)+N d(\bar{y}, \bar{x})+\phi\left(\bar{y}_{\mid n}\right)\right] .
$$

It follows from Lemma 4.5 that for every $M \in \operatorname{Mod}(\mathcal{L}, \mathbb{U})$ and $u \in \mathbb{U}^{k}$

$$
\inf _{g \in \operatorname{Iso}(\mathbb{U})}^{*}\left[\phi^{M}\left(g^{-1} \mathbf{p}_{\mid n}\right)+N d\left(g^{-1} \mathbf{p}_{\mid k}, u\right)\right]=\psi^{M}(u) .
$$

If $n \geq k$ then define $\psi(\bar{x})$ to be the $N$-Lipschitz formula in the $k$ free variables $\bar{x}$

$$
\sup _{m \geq 1} \inf _{y_{0}, \ldots, y_{n-1}}\left[m \tau_{n}\left(\bar{y}, \mathbf{p}_{\mid n}\right)+N d\left(\bar{x}, \bar{y}_{\mid k}\right)+\phi(\bar{y})\right] .
$$

It follows from Lemma 4.6 that for every $M \in \operatorname{Mod}(\mathcal{L}, \mathbb{U})$ and $u \in \mathbb{U}^{k}$

$$
\inf _{g \in \operatorname{Iso}(\mathbb{U})}^{*}\left[\phi^{M}\left(g^{-1} \mathbf{p}_{\mid n}\right)+N d\left(g^{-1} \mathbf{p}_{\mid k}, u\right)\right]=\psi^{M}(u),
$$

which concludes the proof.

§4.3. Linear combinations. The proof of Lemma 4.8 is analogous to the proofs of Lemmas 4.2, 4.5, and 4.6, and it is again omitted.

Lemma 4.8. Suppose that $A, B$ are grey subsets of $\operatorname{Mod}(\mathcal{L}, \mathbb{U}), k, N \in \omega$ with $N \geq 1$, and $\lambda, \mu \in[0,1]$. For any $t \in[0,1], u \in \mathbb{U}^{k}$, and $M \in \operatorname{Mod}(\mathcal{L}, \mathbb{U})$, the following statements are equivalent:

(1) $\inf _{g \in \operatorname{Iso}(\mathbb{U})}^{*}\left[(\lambda A+\mu B)(g M)+N d\left(g^{-1} \mathbf{p}_{\mid k}, u\right)\right]<t$;

(2) There are $\widetilde{k} \geq k$ and $\widetilde{N} \geq N$ and $\widetilde{u} \in \mathcal{O}\left(\mathbf{p}_{\mid \widetilde{k}}\right)$ such that

$$
\begin{aligned}
N d\left(\widetilde{u}_{\mid k}, u\right)+\lambda \sup _{g \in \operatorname{Iso}(\mathbb{U})}^{*}\left[A(g M)-\widetilde{N} d\left(\widetilde{u}, g^{-1} \mathbf{p}_{\mid \widetilde{k}}\right)\right] \\
\\
+\mu \sup _{g \in \operatorname{Iso}(\mathbb{U})}^{*}\left[B(g M)-\widetilde{N} d\left(\widetilde{u}, g^{-1} \mathbf{p}_{\mid \widetilde{k}}\right)\right]<t ;
\end{aligned}
$$


(3) There are $t_{0}<t, \widetilde{k} \geq k, \widetilde{N} \geq \widetilde{N}$ such that for every $m \geq 1$ there is $\widetilde{u} \in \mathbb{U}^{\widetilde{k}}$ such that

$$
\begin{aligned}
& N d\left(\widetilde{u}_{\mid k}, u\right)+\lambda \sup _{g \in \operatorname{Iso}(\mathbb{U})}^{*} {\left[A(g M)-\widetilde{N} d\left(\widetilde{u}, g^{-1} \mathbf{p}_{\mid \widetilde{k}}\right)\right] } \\
&+\mu \sup _{g \in \operatorname{Iso}(\mathbb{U})}^{*}\left[B(g M)-\widetilde{N} d\left(\widetilde{u}, g^{-1} \mathbf{p}_{\mid \widetilde{k}}\right)\right]+m \tau_{\widetilde{k}}\left(\widetilde{u}, \mathbf{p}_{\mid \widetilde{k}}\right)<t_{0} .
\end{aligned}
$$

Lemma 4.9. Suppose that $A, B$ are grey subsets of $\operatorname{Mod}(\mathcal{L}, \mathbb{U})$ that belong to $\mathcal{B}$. If $\lambda, \mu \in$ $[0,1]$, then $\lambda A+\mu B$ belongs to $\mathcal{B}$.

Proof. Since $A, B \in \mathcal{B}$, for every $\widetilde{k}, \widetilde{N} \in \omega$ with $\widetilde{N} \geq 1$ there are $\widetilde{N}$-Lipschitz formulas $\psi_{A, \widetilde{k}, \widetilde{N}}$ and $\psi_{B, \widetilde{k}, \widetilde{N}}$ in $\widetilde{k}$ free variables such that

$$
\sup _{g \in \operatorname{Iso}(\mathbb{U})}^{*} A\left[(g M)-\widetilde{N} d\left(\widetilde{u}, g^{-1} \mathbf{p}_{\mid \widetilde{k}}\right)\right]=\psi_{A, \widetilde{k}, \widetilde{N}}^{M}(\widetilde{u})
$$

and similarly for $B$. Fix $k, N \in \omega$ with $N \geq 1$, and define the $N$-Lipschitz formula $\varphi(\bar{x})$ in the $k$ free variables $\bar{x}$

$$
\inf _{\widetilde{N} \geq N} \inf _{\widetilde{k} \geq k} \sup _{m \geq 1} \inf _{y_{0}, \ldots, y_{\widetilde{k}-1}}\left[m \tau_{\widetilde{k}}\left(\bar{y}, \mathbf{p}_{\mid \widetilde{k}}\right)+N d\left(\bar{y}_{\mid k}, \bar{x}\right)+\lambda \psi_{A, \widetilde{k}, \widetilde{N}}(\bar{y})+\mu \psi_{B, \widetilde{k}, \widetilde{N}}(\bar{y})\right] .
$$

By Lemma 4.8 for $M \in \operatorname{Mod}(\mathcal{L}, \mathbb{U})$ and $u \in \mathbb{U}^{k}$

$$
\text { inf }_{g \in \operatorname{Iso}(\mathbb{U})}^{*}\left[(\lambda A+\mu B)(g M)+N d\left(g^{-1} \mathbf{p}_{\mid k}, u\right)\right]=\varphi^{M}(u) .
$$

In view of Lemma 4.3 this concludes the proof that $\lambda A+\mu B \in \mathcal{B}$.

\section{$\S 4.4$. Infima and suprema.}

Lemma 4.10. If $\left(A_{n}\right)_{n \in \omega}$ is a sequence of grey subsets of $\operatorname{Mod}(\mathcal{L}, \mathbb{U})$ that belong to $\mathcal{B}$, then $\inf _{n} A_{n}$ and $\sup _{n} A_{n}$ belong to $\mathcal{B}$.

Proof. By Lemma 4.4 it is enough to show that $\sup _{n} A_{n} \in \mathcal{B}$. Fix $k, N \in \omega$ with $N \geq 1$. For every $n \in \omega$, since $A_{n} \in \mathcal{B}$ there is an $N$-Lipschitz formula $\varphi_{n}$ such that for every $M \in \operatorname{Mod}(\mathcal{L}, \mathbb{U})$ and $u \in \mathbb{U}^{k}$

$$
\sup _{g \in \operatorname{Iso}(\mathbb{U})}^{*}\left[A_{n}(g M)-N d\left(u, g^{-1} \mathbf{p}_{\mid k}\right)\right]=\varphi_{n}^{M}(u) .
$$

It follows from Proposition 3.1(3) that

$$
\begin{aligned}
\sup _{g \in \operatorname{Iso}(\mathbb{U})}^{*}\left[\sup _{n} A_{n}(g M)-N d\left(u, g^{-1} \mathbf{p}_{\mid k}\right)\right] & =\sup _{n} \sup _{g \in \operatorname{Iso}(\mathbb{U})}^{*}\left[A_{n}(g M)-N d\left(u, g^{-1} \mathbf{p}_{\mid k}\right)\right] \\
& =\left(\sup _{n} \varphi_{n}\right)^{M}(u) .
\end{aligned}
$$

Since $\sup _{n} \varphi_{n}$ is an $N$-Lipschitz formula, this shows that $\sup _{n} A_{n} \in \mathcal{B}$.

\section{REFERENCES}

[BK] H. Becker and A. S. Kechris, The Descriptive Set Theory of Polish Group Actions, London Mathematical Society Lecture Note Series, vol. 232, Cambridge University Press, Cambridge, 1996. 
[BYBHU] I. Ben Yaacov, A. Berenstein, C. W. Henson, and A. Usvyatsov, Model theory for metric structures, Model Theory with Applications to Algebra and Analysis. Vol. 2, 2008, pp. 315-427.

[BYI] I. Ben Yaacov and J. Iovino, Model theoretic forcing in analysis, Annals of Pure and Applied Logic 158 (2009), no. 3, 163-174.

[BYM] I. Ben Yaacov and J. Melleray, Grey subsets of Polish spaces, Journal of Symbolic Logic (to appear).

[BYNT] I. Ben Yaacov, A. Nies, and T. Tsankov, A Lopez-Escobar theorem for continuous logic, arXiv:1407.7102 (2014).

[E] C. J. Eagle, Omitting types for infinitary [0,1]-valued logic, Annals of Pure and Applied Logic 165 (2014), no. 3, 913-932.

$\left[\mathrm{EFP}^{+}\right]$G. A. Elliott, I. Farah, V. I. Paulsen, C. Rosendal, A. S. Toms, and A. Törnquist, The isomorphism relation for separable $C^{*}$-algebras, Mathematical Research Letters 20 (2013), no. 6, 1071-1080.

[G] S. Gao, Invariant Descriptive Set Theory, Pure and Applied Mathematics (Boca Raton), vol. 293, CRC Press, Boca Raton, FL, 2009.

[IMI] A. Ivanov and B. Majcher-Iwanow, Polish G-spaces and continuous logic, arXiv:1304.5135 (2013).

[K] A. S. Kechris, Classical Descriptive Set Theory, Graduate Texts in Mathematics, vol. 156, Springer-Verlag, New York, 1995.

[LE] E. G. K. Lopez-Escobar, An interpolation theorem for denumerably long formulas, Fundamenta Mathematicae 57 (1965), 253-272.

[M1] G. W. Mackey, Borel structure in groups and their duals, Transactions of the American Mathematical Society 85 (1957), no. 1, 134-165.

[M2] J. Melleray, On the geometry of Urysohn's universal metric space, Topology and its Applications 154 (2007), no. 2, 384-403.

[M3] _ Some geometric and dynamical properties of the Urysohn space, Topology and its Applications 155 (2008), no. 14, 1531-1560.

[P] P. Petersen, Riemannian geometry, Second, Graduate Texts in Mathematics, vol. 171, Springer, New York, 2006.

[S] N. Sequeira, Infinitary Continuous Model Theory, Master's Thesis, 2013.

[T] H. Tuuri, Relative separation theorems for $\mathcal{L}_{\kappa+\kappa}$., Notre Dame Journal of Formal Logic 33 (1992), no. 3, 383-401.

[U1] V. V. Uspenskij, A universal topological group with a countable basis, Functional Analysis and its Applications 20 (1986), no. 2, 160-161.

[U2] - On the group of isometries of the Urysohn universal metric space, Commentationes Mathematicae Universitatis Carolinae 31 (1990), no. 1, 181-182.

[V] R. Vaught, Invariant sets in topology and logic, Fundamenta Mathematicae 82 (1974), 269-294.

Samuel Coskey, Department of Mathematics, Boise State University, 1910 University Dr., BoISE ID 83725-1555

E-mail address: scoskey@nylogic.org

$U R L$ : boolesrings.org/scoskey

Fakultät für Mathematik, Universität Wien, Oskar-Morgenstern-Platz 1, Room 02.126, 1090 Wien, Austria.

E-mail address: martino.lupini@univie.ac.at

$U R L$ : www.lupini.org 